\title{
Epidemiology and clinical impact of viral, atypical, and fungal respiratory pathogens in symptomatic immunocompromised patients: a two-center study using a multi-parameter customized respiratory Taqman ${ }^{\circledast}$ array card
}

\author{
Deborah Steensels $^{1}$ (D) $\cdot$ Marijke Reynders $^{2} \cdot$ Patrick Descheemaeker $^{2} \cdot$ Martin D. Curran $^{3} \cdot$ Maya Hites $^{4}$. \\ Isabelle Etienne ${ }^{5} \cdot$ Isabel Montesinos ${ }^{1}$
}

Received: 17 March 2019 / Accepted: 1 May 2019 / Published online: 20 May 2019

(C) Springer-Verlag GmbH Germany, part of Springer Nature 2019

\begin{abstract}
The prevalence of respiratory viruses in immunocompromised adult patients and the association with clinical outcomes is still underexplored. Our goal was to assess the epidemiology and the potential clinical impact of respiratory viral infections in a highrisk patient population. Two large hospitals performed a respiratory Taqman array card (TAC), targeting 24 viruses, 8 bacteria, and 2 fungi simultaneously, on 435 samples from 397 symptomatic immunocompromised patients. Clinical details were collected retrospectively using a structured case report form. An overall positivity rate of $68 \%$ was found (51\% mono- and $17 \%$ co-infections). Pathogen distribution was as follows: influenza A (20.7\%), rhinoviruses (15.2\%), coronaviruses (7.8\%), Pneumocystis jirovecii (7.4\%), RSV (7.1\%), and CMV (6.0\%) were the most frequently encountered, followed by HSV (5.5\%), hMPV (4.4\%), parainfluenza viruses (3.9\%), influenza B (3.7\%), and Aspergillus species (3.7\%). Other pathogens were not detected or detected only in $\leq 1 \%$ of samples. Hospital and ICU admission rates were $84 \%$ and $11 \%$, respectively. The presence of a pathogen was strongly associated with higher need for supplemental oxygen $(p=0.001)$, but it had no impact on ICU admission, mechanical ventilation requirement, antibacterial therapy, or mortality. In conclusion, our study described the epidemiology of respiratory pathogens in a large group of symptomatic immunocompromised patients and provides evidence of a relationship between pathogen detection and the need for supplemental oxygen. This association was still found after the exclusion of the results positive for influenza viruses, suggesting that non-influenza viruses contribute to severe respiratory illness in patients with compromised immunity.
\end{abstract}

Keywords Respiratory tract infections $\cdot$ Immunocompromised host $\cdot$ Epidemiology $\cdot$ Clinical impact

Deborah Steensels

deborah.steensels@zol.be

1 Department of Clinical Microbiology, CUB-Erasme, Université Libre de Bruxelles, Brussels, Belgium

2 Department of Clinical Microbiology, AZ St-Jan Brugge-Oostende Hospital, Bruges, Belgium

3 Public Health England, Clinical Microbiology and Public Health Laboratory, Addenbrooke's Hospital, Cambridge, UK

4 Department of Infectious Diseases, CUB-Erasme, Université Libre de Bruxelles, Brussels, Belgium

5 Department of Pulmonology - Pulmonary Transplant, CUB-Erasme, Université Libre de Bruxelles, Brussels, Belgium

\section{Introduction}

The major impact of respiratory virus infections in patients with hematologic malignancies, hematopoietic stem cell transplantation, and solid organ transplantation has been recognized over the last decades [1-4].

In most immunocompromised populations, respiratory viruses are responsible for lower respiratory tract infections, associated with a significant morbidity and mortality. The detection of viral RNA in a respiratory sample suggests a viral contribution to respiratory symptomatology and pathology. This issue is of particular importance in immunocompromised patients who may be infected with several agents and who are prone to bacterial superinfections. In addition to respiratory viruses, the impact of so-called atypical bacteria, such as Mycoplasma pneumoniae, Bordetella pertussis, Chlamydophila 
pneumoniae, and Legionella pneumophila and fungi such as Pneumocystis jirovecii and Aspergillus species, in immunocompromised hosts also needs to be established in large studies.

The primary goal of the present study is to describe the epidemiology of respiratory pathogens in a large two-centric group of immunocompromised patients with symptoms of an upper or lower respiratory tract infection using a multiparameter customized respiratory Taqman ${ }^{\circledR}$ array card, targeting 24 viruses, 8 bacteria, and 2 fungi simultaneously [5-7]. In addition, secondary goals are to investigate the possible association of pathogen detection (presence versus absence, mono- versus co-infections) with key clinical parameters.

\section{Material and methods}

\section{Patients and samples}

A hospital-based retrospective cohort study was conducted at two tertiary care hospitals, the academic Erasme Hospital (Brussels) and the general St-Jan Hospital (Bruges-Ostend) with an in-between distance of about $95 \mathrm{~km}$. All adult immunocompromised patients with symptoms of an upper or lower respiratory tract infection that underwent testing for respiratory pathogens were retrospectively included from December 2014 to June 2015 (6-month period) at the St-Jan Hospital and from December 2014 to December 2015 (1-year period) for patients at the Erasme Hospital.

Patients had at least one of the following acute respiratory symptoms, often associated with fever: rhinorrhea, cough, sputum, dyspnea, unexplained lung function decline, or new chest X-ray abnormalities.

Patient samples were excluded when respiratory symptoms started $\geq 14$ days before the first sample collection. Samples from different respiratory episodes were included when the episodes were separated with at least 1 month. Patients should have been free from respiratory infectious symptoms between separated episodes.

Immunocompromised status was defined as presence of a disease and/or treatment known to impair the immune system, such as solid organ transplant (SOT) under immunosuppressive therapy (group 1), solid (group 2) or hematological malignancy (group 3) under chemotherapy, or other underlying disease needing long-term high-dose corticosteroid therapy or immunosuppressive therapy (group 4). Patients infected with HIV with a CD4 count $<200$ / $\mathrm{mm}^{3}$ were also included (group 4).

\section{Data collection}

The following clinical details were collected retrospectively using a structured case report form: age, gender, underlying disease, influenza vaccination status, admission unit, date of hospitalization and length of stay, ICU admission, need for supplemental oxygen therapy and/or mechanical ventilation, antiviral and/or antibacterial treatment and survival.

Inclusion and collection of clinical data were performed by one microbiologist from each hospital.

\section{Nucleic acid extraction and TAC testing}

Patient samples were prospectively tested at the St-Jan Hospital and retrospectively tested at the Erasme Hospital with a customized TAC respiratory panel (v8.0 and v10.0 premarket version Cambridge-Bruges, respectively) which included testing for the pathogens described previously [5]. It is important to note that the assay for herpes simplex virus 1 and 2 was not yet present on version 8.0 of the cards and was thus not included for the samples from St-Jan Hospital. The detailed procedure was described previously [5].

\section{Statistical analysis}

Data were presented for the entire study group and stratified by underlying conditions to assess potential differences in patient characteristics regarding the epidemiology of respiratory pathogens and key clinical parameters.

Data were analyzed using GraphPad (GraphPad Software Inc., San Diego, CA, USA) and Stata/IC 15.1. Pearson's chisquared test and Fisher's exact test (when appropriate) were used to compare positivity rates and outcome between the different populations. The Mann-Whitney-Wilcoxon test was used to compare non-Gaussian distribution variables. Multivariate logistic regression analysis was performed on all samples and on samples without influenza infection to identify the clinical characteristics independently associated with the presence of pathogen(s). All clinical characteristics were included in the models except the length of stay (collinearity with hospitalization). The goodness-of-fit (HosmerLemeshow) test was used to verify if the model adjusted appropriately to the data. Results with a $p<0.05$ were considered significant.

\section{Results}

\section{Patients}

Four hundred-thirty five samples were obtained from $397 \mathrm{im}$ munocompromised patients. Baseline patient characteristics are shown in Table 1. Of group 3 patients who underwent allo- or auto-hematological stem cell transplantation (HSCT), 64.3\% received the transplantation less than 100 days before their respiratory episode and therefore inclusion. 
Table 1 Baseline patient characteristics

\begin{tabular}{|c|c|c|c|}
\hline Characteristic & Erasme Hospital & St-Jan Brugge-Oostende Hospital & All patients \\
\hline Age, median (range), years & $58(18,85)$ & $67(16,96)$ & $62(16,96)$ \\
\hline Gender, male, no. (\%) & $119(54.3)$ & $95(53.4)$ & $214(53.9)$ \\
\hline \multicolumn{4}{|l|}{ Underlying condition, no. (\%) } \\
\hline -Solid organ transplantation (group 1) & $118(53.9)$ & $12(6.7)$ & $130(33.0)$ \\
\hline •Pulmonary & $50(22.8)$ & $0(0.0)$ & $50(12.7)$ \\
\hline •Renal & $34(15.5)$ & $10(5.6)$ & $44(11.2)$ \\
\hline •Hepatic & $16(7.3)$ & $2(1.1)$ & $18(4.5)$ \\
\hline -Cardiac & $9(4.1)$ & $0(0.0)$ & $9(2.3)$ \\
\hline •Combined* & $9(4.1)$ & $0(0.0)$ & $9(2.3)$ \\
\hline -Solid malignancy (group 2) & $38(17.4)$ & $24(13.5)$ & $62(15.7)$ \\
\hline -Hematological disorders (group 3) & $37(16.9)$ & $112(62.9)$ & $149(37.8)$ \\
\hline •Hematological malignancy & $26(11.9)$ & $101(56.7)$ & $127(32.2)$ \\
\hline •AlloHSCT & $3(1.4)$ & $7(3.9)$ & $10(2.5)$ \\
\hline •AutoHSCT & $8(3.7)$ & $4(2.2)$ & $12(3.0)$ \\
\hline $\begin{array}{l}\text {-Other underlying disease needing long-term corticosteroids } \\
\text { therapy or immunosuppressive therapy, or HIV CD } 4<200 / \mathrm{mm}^{3} \text { (group 4) }\end{array}$ & $26(13.2)$ & $30(16.9)$ & $56(14.1)$ \\
\hline
\end{tabular}

*Lung + kidney $(n=2)$, lung + heart $(n=4)$, kidney + heart $(n=1)$, kidney + liver $(n=1)$, lung + kidney + heart $(n=1)$

In total, $334(76.8 \%)$ nasopharyngeal swabs in Universal Transport Medium (UTM-RT, Copan Diagnostics, CA, USA) and $101(23.2 \%)$ bronchoalveolar lavage (BAL) samples were included.

Overall, patients were hospitalized for $83.9 \%$ of respiratory episodes, with an $11.1 \%$ admission rate to the intensive care unit. Among the 397 patients, 9 male (4.2\%) and 10 female patients $(5.5 \%)$ died within 15 days of their first respiratory episode (difference not significant, $p=0.56$ ) and 14 male $(6.5 \%)$ and 14 female patients $(7.7 \%)$ died within 30 days of their first respiratory episode (difference not significant, $p=$ $0.67)$. The mortality rate within 30 days of the first respiratory episode was significantly higher in patients older than 65 years old $(p=0.03)$.

For nearly one third (32.0\%) of all patients, medical records did not contain information on influenza vaccination. When taking into account only those patients for which the influenza vaccination status was known, the vaccination rates did not differ between study groups and amount to an overall coverage of $70.7 \%$.

\section{Respiratory pathogen analysis}

The overall positivity rate of the TAC was $68 \%$, with $51 \%$ positive for one pathogen and $17 \%$ for more than one pathogen. Positivity rate was not significantly different between nasopharyngeal swabs and BAL samples. After the correction for multiple comparisons, the positivity rate of TAC in group 3 (hematologic disorders) (59.9\%) was significantly lower than that in group $4(80.3 \%)(p=0.02)$.
The number of samples positive with more than one pathogen did not differ significantly between groups.

Overall, the pathogen distribution at genus level was as follows: influenza A $(20.7 \%)$, rhinoviruses $(15.2 \%)$, coronaviruses $(7.8 \%)$, P. jirovecii $(7.4 \%)$, RSV (7.1\%), and CMV (6.2\%) were the most frequently encountered, followed by HSV (5.5\%), hMPV (4.4\%), parainfluenza viruses $(3.9 \%)$, influenza B $(3.7 \%)$, and Aspergillus species $(3.7 \%)$. Adenovirus, enterovirus, bocavirus, L. pneumophila, M. pneumoniae, B. pertussis, and $B$. parapertussis were detected in only a limited number of samples $(\leq 1 \%)$. Those most frequently involved in coinfections were rhinovirus $(n=29)$, influenza virus $(n=$ 26), CMV ( $n=19)$, $P$. jirovecii $(n=18)$, HSV $(n=17)$, and coronavirus $(n=14)$. Each sample positive for an atypical bacterial pathogen was as well positive for one or more viral pathogens. Detailed results are shown in Table 2 and Fig. 1. Nasopharyngeal swabs had a significantly higher positivity rate for influenza $\mathrm{A}(p<0.001)$ and coronaviruses $(p=$ 0.01 ), while bronchoalveolar lavage samples were more frequently positive for HSV $(p=0.03), \mathrm{CMV}(p<0.001)$, $P$. jirovecii $(p=0.001)$, and Aspergillus species $(p<0.001)$. The median $\mathrm{Ct}$ values (range) for these opportunistic pathogens in BAL samples were Ct 31 (21-35) for HSV, Ct 31 (23-35) for CMV, Ct 29 (21-35) for P. jirovecii, and Ct 32 (19-38) for Aspergillus species.

\section{Clinical features according to TAC results}

Retrospectively gathered clinical parameters were compared for patients with a positive versus a negative TAC result 
(Table 3). The length of stay was not always restricted to respiratory episode but was in some cases much longer due to for example other co-morbidities, chemotherapy cures, other infectious syndromes, or even long delay for transfer to rehabilitation centers. The administration of antibiotics and/ or antivirals was very often started empirically for treatment of the respiratory infection, but some patients were also on prophylactic drugs. Patients on antiviral therapy were most frequently treated with oseltamivir $(68 \%)$, then acyclovir (18\%), (val)ganciclovir (10\%), and ribavirin $(3 \%)$.

Overall, patients for whom a respiratory pathogen was found were significantly more likely to require supplemental oxygen $(40.6 \%$ vs $23.9 \%(p=0.001))$ and to be treated with antivirals $(41.9 \%$ vs $10.3 \%(p<0.001))$, but there was no impact on ICU admission, mechanical ventilation requirement, antibacterial therapy, or mortality. After excluding all patients with a positive result for influenza A or B viruses, these findings did not change. Table 3 shows detailed results for all patient episodes and per patient group. Only for group 4 , patients that were hospitalized were more likely to be positive for a respiratory pathogen $(p=0.02)$. Multivariate analysis showed that, adjusted for the presence of other predictors in the model, the administration of supplemental oxygen and antiviral therapy was still statistically associated with the presence of pathogens $(p<0.001)$.

The same clinical parameters were compared for patients with one single pathogen found by TAC versus patients positive for more than one pathogen (Table 3). There were no significant differences between these groups.

\section{Discussion}

Solid organ transplantation and hematologic oncology are part of the main activities of the Erasme and St-Jan Hospital, respectively. This study included nearly 400 immunocompromised patients who presented to the hospital with symptoms of a respiratory infection.

The performances of the respiratory TAC used in this study were described previously $[5,6]$.

The overall positivity rate was relatively high $(68 \%)$, as expected when including only symptomatic patients.

Influenza A virus was the most frequently encountered pathogen. The proportion of flu infections in immunocompromised patients described in the literature varies due to the variable nature of the influenza virus itself and its ability to
Table 2 Pathogen distribution (number and percentage), overall and for each patient group

\begin{tabular}{|c|c|c|c|c|c|c|c|c|c|c|}
\hline \multirow[t]{2}{*}{ TAC } & \multirow{2}{*}{$\begin{array}{l}\text { Overall } \\
\text { No. }\end{array}$} & \multirow[b]{2}{*}{$\%$} & \multicolumn{2}{|l|}{ Group 1} & \multicolumn{2}{|l|}{ Group 2} & \multicolumn{2}{|c|}{ Group 3} & \multicolumn{2}{|c|}{ Group 4} \\
\hline & & & No. & $\%$ & No. & $\%$ & No. & $\%$ & No. & $\%$ \\
\hline Influenza A (a) & 90 & 20.7 & 23 & 14.9 & 18 & 28.6 & 33 & 21.0 & 16 & 26.2 \\
\hline Influenza B & 16 & 3.7 & 8 & 5.2 & 1 & 1.6 & 6 & 3.8 & 1 & 1.6 \\
\hline RSV (b) & 31 & 7.1 & 11 & 7.1 & 5 & 7.9 & 11 & 7.0 & 4 & 6.6 \\
\hline hMPV & 19 & 4.4 & 11 & 7.1 & 1 & 1.6 & 5 & 3.2 & 2 & 3.3 \\
\hline Adenovirus & 5 & 1.1 & 3 & 1.9 & 0 & 0.0 & 1 & 0.6 & 1 & 1.6 \\
\hline Enterovirus & 1 & 0.2 & 0 & 0.0 & 0 & 0.0 & 0 & 0.0 & 1 & 1.6 \\
\hline $\begin{array}{l}\text { Parainfluenza } \\
\text { virus (c) }\end{array}$ & 17 & 3.9 & 9 & 5.8 & 2 & 3.2 & 3 & 1.9 & 3 & 4.9 \\
\hline Rhinovirus & 66 & 15.2 & 30 & 19.5 & 3 & 4.8 & 22 & 14.0 & 11 & 18.0 \\
\hline Coronavirus (d) & 34 & 7.8 & 7 & 4.5 & 6 & 9.5 & 18 & 11.5 & 3 & 4.9 \\
\hline Bocavirus & 1 & 0.2 & 1 & 0.6 & 0 & 0.0 & 0 & 0.0 & 0 & 0.0 \\
\hline $\mathrm{HSV} 1+2$ & 24 & 5.5 & 13 & 8.4 & 5 & 7.9 & 2 & 1.3 & 4 & 6.6 \\
\hline CMV & 27 & 6.2 & 18 & 11.7 & 3 & 4.8 & 1 & 0.6 & 5 & 8.2 \\
\hline B. pertussis & 1 & 0.2 & 0 & 0.0 & 0 & 0.0 & 1 & 0.6 & 0 & 0.0 \\
\hline B. parapertussis & 1 & 0.2 & 1 & 0.6 & 0 & 0.0 & 0 & 0.0 & 0 & 0.0 \\
\hline L. pneumophila & 3 & 0.7 & 0 & 0.0 & 1 & 1.6 & 1 & 0.6 & 1 & 1.6 \\
\hline M. pneumoniae & 2 & 0.5 & 2 & 1.3 & 0 & 0.0 & 0 & 0.0 & 0 & 0.0 \\
\hline P. jirovecii & 32 & 7.4 & 10 & 6.5 & 6 & 9.5 & 7 & 4.5 & 9 & 14.8 \\
\hline $\begin{array}{l}\text { Aspergillus } \\
\text { species }\end{array}$ & 16 & 3.7 & 10 & 6.5 & 1 & 1.6 & 3 & 1.9 & 2 & 3.3 \\
\hline
\end{tabular}

(a) H1-2009: $n=11, \mathrm{H} 3$ seasonal: $n=77$, non-typed: $n=2$

(b) RSV A: $n=25$, RSV B: $n=5$, non-typed: $n=1$

(c) PIV1: $n=5$, PIV2: $n=1$, PIV3: $n=9$, PIV4: $n=2$

(d) CoV 229E: $n=16$, CoV OC43: $n=8, \operatorname{CoV}$ NL63: $n=6$, CoV HKU-1: $n=4$ 
Fig. 1 TAC results per patient group (\% of total number of samples)

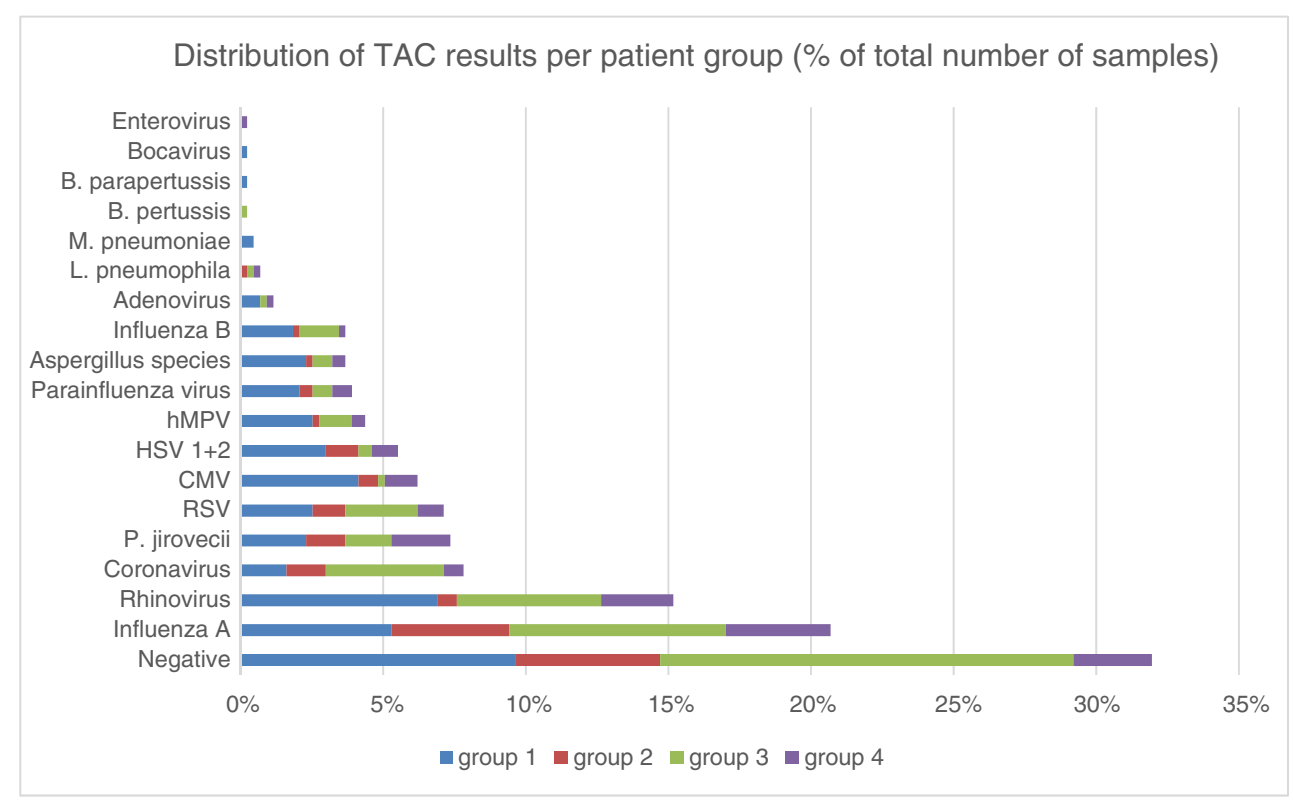

cause annual outbreaks of different intensity. The influenza epidemic during the study period was of high intensity and long duration in Belgium [8]. Overall, 43.9\% of samples were positive for non-influenza viruses. The so-called "common cold" viruses (rhinovirus and coronavirus) were frequently encountered. This reflects the epidemiology of the adult population living in the community and is in line with findings described in the literature [9-12]. In addition to the low prevalence of "atypical" bacterial pathogens, which might be explained by the exclusion of samples taken $\geq 14$ days after the onset of symptoms, viruses such as bocavirus, adenovirus, and enterovirus were very infrequent in this adult population $(<1 \%)$.

This study showed a relatively high incidence of opportunistic pathogens in BAL samples. The HSV positivity rate might be underestimated since this target was not present on the TAC used at the St-Jan Brugge-Oostende Hospital. The median Ct value was 29 for $P$. jirovecii, indicating a significant pathogen load. Nearly all patients with a positive result for $P$. jirovecii in BAL were treated with high-dose cotrimoxazole during at least 21 days. Some data exist on the epidemiology and impact of opportunistic pathogens such as $P$. jirovecii [13-15], CMV [16, 17], HSV [18-20], and Aspergillus species [21] in immunocompromised patients. Quantitative assays or working with (arbitrary) cut-off $\mathrm{Ct}$ values seems critical to determine clinical relevance, although every positive result in a BAL sample could be of clinical importance, especially in this group of patients. $\mathrm{Ct}$ values above ct 28 for Aspergillus species or Pneumocystis may point to a very early phase of reactivation but on the other hand they may correspond to colonization (or contamination).

When comparing the two main patient groups, hematologic oncology patients had a lower TAC positivity rate than SOT patients and were less likely to have a co-infection, although these associations were not statistically significant. The observed differences might be explained by differences in type and level of immunosuppression. Interestingly, influenza A virus and coronaviruses were more common in hematologic oncology patients than in the SOT patients. These rates might have been biased due to the shorter inclusion period in St-Jan Hospital covering the influenza epidemics (but not the RSV peak), where most of these hematology patients were included. Since the two hospitals had a different inclusion profile and since they are geographically separated, this might also have biased the differences in pathogen prevalence between patient groups. However, the two centers are only $95 \mathrm{~km}$ from each other, and the climate is similar in both regions.

Patients who tested positive for a respiratory pathogen were more likely to be treated with supplemental oxygen and antiviral therapy. The clinical impact of influenza virus infections is well known, but even when all patients positive for influenza viruses were excluded from analysis, the presence of a respiratory pathogen was still associated with need for supplemental oxygen and antiviral treatment. Other studies also revealed a significant association between a positive respiratory virus PCR and the presence of an acute respiratory disease [8, 22-24]. In our study however, the association with antiviral therapy may be confounded since the treating clinicians were not blinded to results. At the Erasme Hospital, TAC analysis was performed retrospectively, but the clinicians were aware of conventional viral testing results (based on direct fluorescent antibody testing and viral culture) [3]. At the St-Jan Hospital, clinicians received the results of TAC analysis within $24 \mathrm{~h}$ after sampling.

There are some limitations to this study. The inclusion period was 6 months longer at the Erasme Hospital. However, 


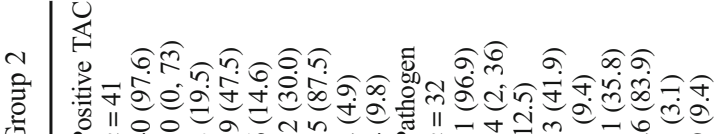

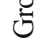

言

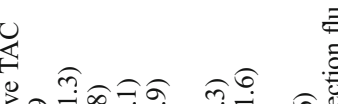

येक्ष

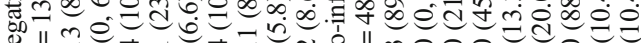

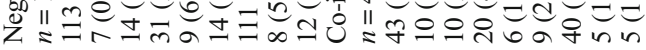
in

$\frac{2}{4}$

$\stackrel{\circ}{\frac{m}{2}}$

茕

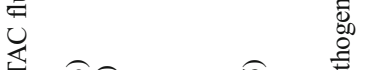

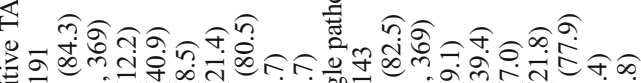
药

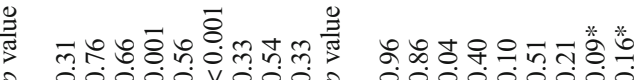
cos 药

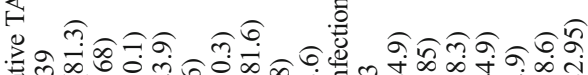

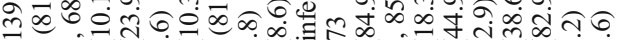

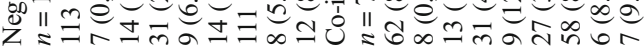
告

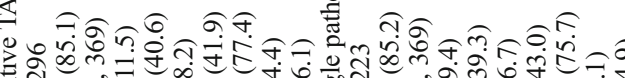

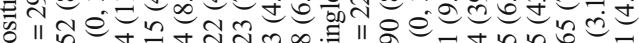

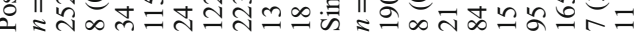
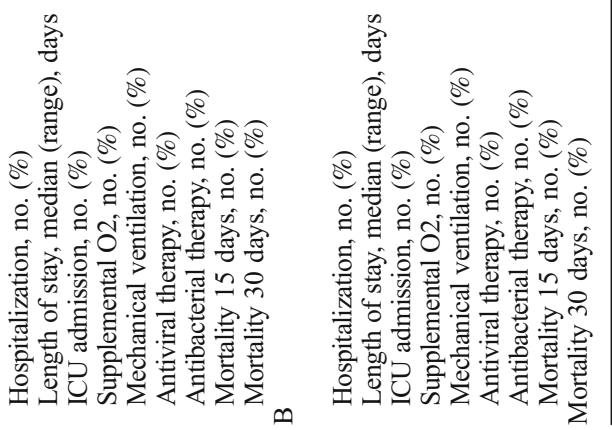

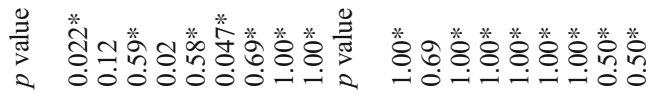

$\frac{1}{5}$

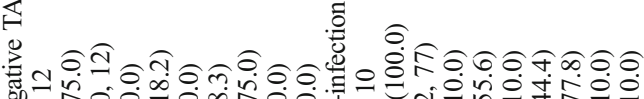
ż II

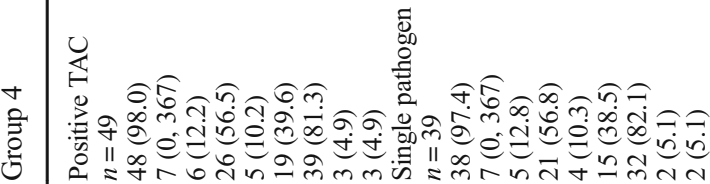

疍 a 00000v000 0.0000000

I

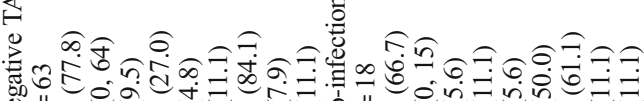

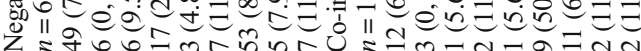

过

हैं

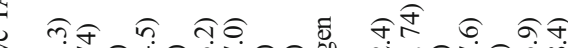

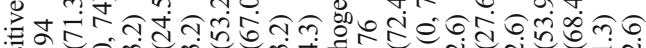
: " 6 mm

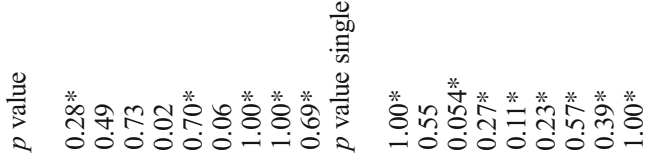
$\stackrel{4}{r}$

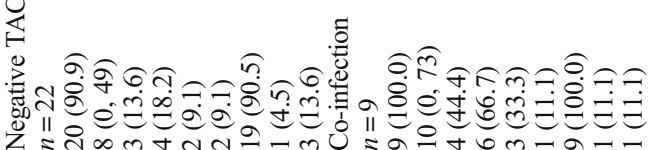
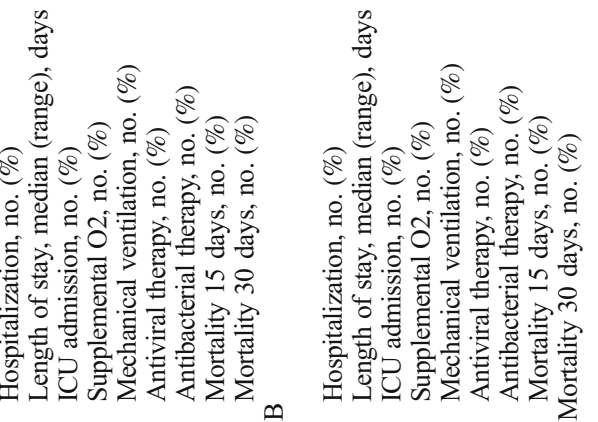
since winter and spring seasons were covered by both hospitals, only $24 \%$ of all samples were included at the Erasme Hospital in this extra 6-month period.

Cultured bacteria as potential causal pathogens of respiratory infections were not taken into account and might have biased the results for clinical impact of the tested respiratory pathogens and mono- versus co-infection.

In conclusion, our study described the epidemiology of respiratory viruses, atypical bacteria, and fungi in a large group of symptomatic immunocompromised patients. Our analysis provides an evidence of a relationship between pathogen detection and the need for supplemental oxygen. This association was no different after the exclusion of results positive for influenza viruses; non-influenza viruses appear to contribute to severe respiratory disease in patients with compromised immunity.

Acknowledgments We thank the technicians of both virology and molecular microbiology laboratories for their technical assistance during this study.

\section{Compliance with ethical standards}

Conflict of interest The authors declare that they have no conflict of interest.

Ethical approval This study was approved by the ethical committee of the Erasme and St-Jan Hospital.

Informed consent Not applicable.

\section{References}

1. Chemaly RF, Shah DP, Boeckh MJ (2014) Management of respiratory viral infections in hematopoietic cell transplant recipients and patients with hematologic malignancies. Clin Infect Dis 59:S344S351

2. Vakil E, Evans SE (2017) Viral pneumonia in patients with hematologic malignancy or hematopoietic stem cell transplantion. Clin Chest Med 38:97-111

3. Abbas S, Raybould JE, Sastry S, de la Cruz O (2017) Respiratory viruses in transplant recipients: more than just a cold. Clinical syndromes and infection prevention principles. Int J Infect Dis 62:8693

4. Magnusson J, Westin J, Andersson LM, Brittain-Long R, Riise GC (2013) The impact of viral respiratory tract infections on long-term morbidity and mortality following lung transplantation: a retrospective cohort study using a multiplex PCR panel. Transplantation 95(2):383-388

5. Steensels D, Reynders M, Descheemaeker P, Curran MD, Jacobs F, Denis O, Delforge M-L, Montesinos I (2015) Clinical evaluation of a multi-parameter customized respiratory TaqMan® array card compared to conventional methods in immunocompromised patients. J Clin Virol 72:36-41

6. Steensels D, Reynders M, Descheemaeker P, Curran MD, Jacobs F, Denis O, Delforge M-L, Montesinos I (2017) Performance evaluation of direct fluorescent antibody, Focus Diagnostics Simplexa ${ }^{\mathrm{TM}}$ Flu A/B \& RSV and multi-parameter customized respiratory
Taqman ${ }^{\circledR}$ array card in immunocompromised patients. J Virol Methods 245:61-65

7. Steensels D, Delforge M-L, Lagrou K, Beuselinck K, Montesinos I (2017) Development of a customized TaqMan ${ }^{\circledR}$ Array card for simultaneous detection of 32 respiratory pathogens. Adv Techn Clin Microbiol 2:1-8

8. National influenza Centre, Scientific Institute of Public Health. Virological surveillance of influenza in Belgium: season 20142015. Reference number: D/2015/2505/60

9. Drieghe S, Ryckaert I, Beuselinck K, Lagrou K, Padalko E (2014) Epidemiology of respiratory viruses in bronchoalveolar lavage samples in a tertiary hospital. J Clin Virol 59:208-211

10. Garbino J, Soccal PM, Aubert JD, Rochat T, Meylan P, Thomas Y et al (2009) Respiratory viruses in bronchoalveolar lavage: a hospital-based cohort study in adults. Thorax 64:399-404

11. Manuel O, López-Medrano F, Keiser L, Welte T, Carratalà J, Cordero E, Hirsch HH (2014) ESCMID Study Group of Infection in Compromised Hosts (ESGICH). Influenza and other respiratory virus infections in solid organ transplant recipients. Clin Microbiol Infect 20:102-108

12. Weigt SS, Gregson AL, Deng JC, Lynch JP 3rd, Belperio JA (2011) Respiratory viral infections in hematopoietic stem cell and solid organ transplant recipients. Semin Respir Crit Care Med 32:471493

13. Tasaka S, Tokuda H (2012) Pneumocystis jirovecii pneumonia in non-HIV-infected patients in the era of novel immunosuppressive therapies. J Infect Chemother 18:793-806

14. Cordonnier C, Cesaro S, Maschmeyer G, Einsele H, Donnelly JP, Alanio A, Hauser PM, Lagrou K, Melchers WJ, Helweg-Larsen J, Matos O, Bretagne S, Maertens J, Fifth European Conference on Infections in Leukemia (ECIL-5), a joint venture of The European Group for Blood and Marrow Transplantation (EBMT), The European Organization for Research and Treatment of Cancer (EORTC), the immunocompromised host society (ICHS) and the European LeukemiaNet (ELN) (2016) Pneumocystis jirovecii pneumonia: still a concern in patients with haematological malignancies and stem cell transplant recipients. J Antimicrob Chemother 71:2379-2385

15. Lee HY, Kang HS, Lee HY, Rhee CK, Lee SY, Kim SC, Kim SJ, Park YJ, Kim YK, Kang JY (2017) Clinical significance of positive Pneumocystis jirovecii polymerase chain reaction in non-human immunodeficiency virus immunocompromised patients in a real practice. Korean J Intern Med 32:478-485

16. Königshausen E, Hengel H, Adams O, Bry H, Steiner S, Priegnitz C, Hansen A, Sellin L, Rump LC, Voiculescu A (2016) Pulmonary cytomegalovirus replication in renal transplant patients with late onset pneumonitis. Ann Transplant 21:235-240

17. Santos CA, Brennan DC, Yusen RD, Olsen MA (2015) Incidence, risk factors and outcomes of delayed-onset cytomegalovirus disease in a large retrospective cohort of lung transplant recipients. Transplantation 99:1658-1666

18. Aisenberg GM, Torres HA, Tarrand J, Safdar A, Bodey G, Chemaly RF (2009) Herpes simplex virus lower respiratory tract infection in patients with solid tumors. Cancer 115:199-206

19. Simoons-Smit AM, Kraan EM, Beishuizen A, Strack van Schijndel RJ, Vandenbroucke-Grauls CM (2006) Herpes simplex virus type 1 and respiratory disease in critically-ill patients: real pathogen or innocent bystander? Clin Microbiol Infect 12:1050-1059

20. De Vos N, Van Hoovels L, Vankeerberghen A, Van Vaerenbergh K, Boel A, Demeyer I, Creemers L, De Beenhouwer H (2009) Monitoring of herpes simplex virus in the lower respiratory tract of critically ill patients using real-time PCR: a prospective study. Clin Microbiol Infect 15:358-363

21. Kosmidis C, Denning DW (2015) The clinical spectrum of pulmonary aspergillosis. Thorax 70:270-277 
22. Garbino J, Gerbase MW, Wunderli W, Deffernez C, Thomas Y, Rochat T, Ninet B, Schrenzel J, Yerly S, Perrin L, Soccal PM, Nicod L, Kaiser L (2004) Lower respiratory viral illnesses: improved diagnosis by molecular methods and clinical impact. Am J Respir Crit Care Med 170:1197-1203

23. Sundell N, Andersson LM, Brittain-Long R, Sundvall PD, Alsiö A,, Lindh M, Gustavsson L, Westin J (2019) PCR detection of respiratory pathogens in asymptomatic and symptomatic adults. J Clin Microbiol 57:e00716-18
24. Walker, Ison (2014) Respiratory viral infections among hospitalized adults: experience of a single tertiary healthcare hospital. Influenza Other Respir Viruses 8:282-292

Publisher's note Springer Nature remains neutral with regard to jurisdictional claims in published maps and institutional affiliations. 